Provided for non-commercial research and education use. Not for reproduction, distribution or commercial use.

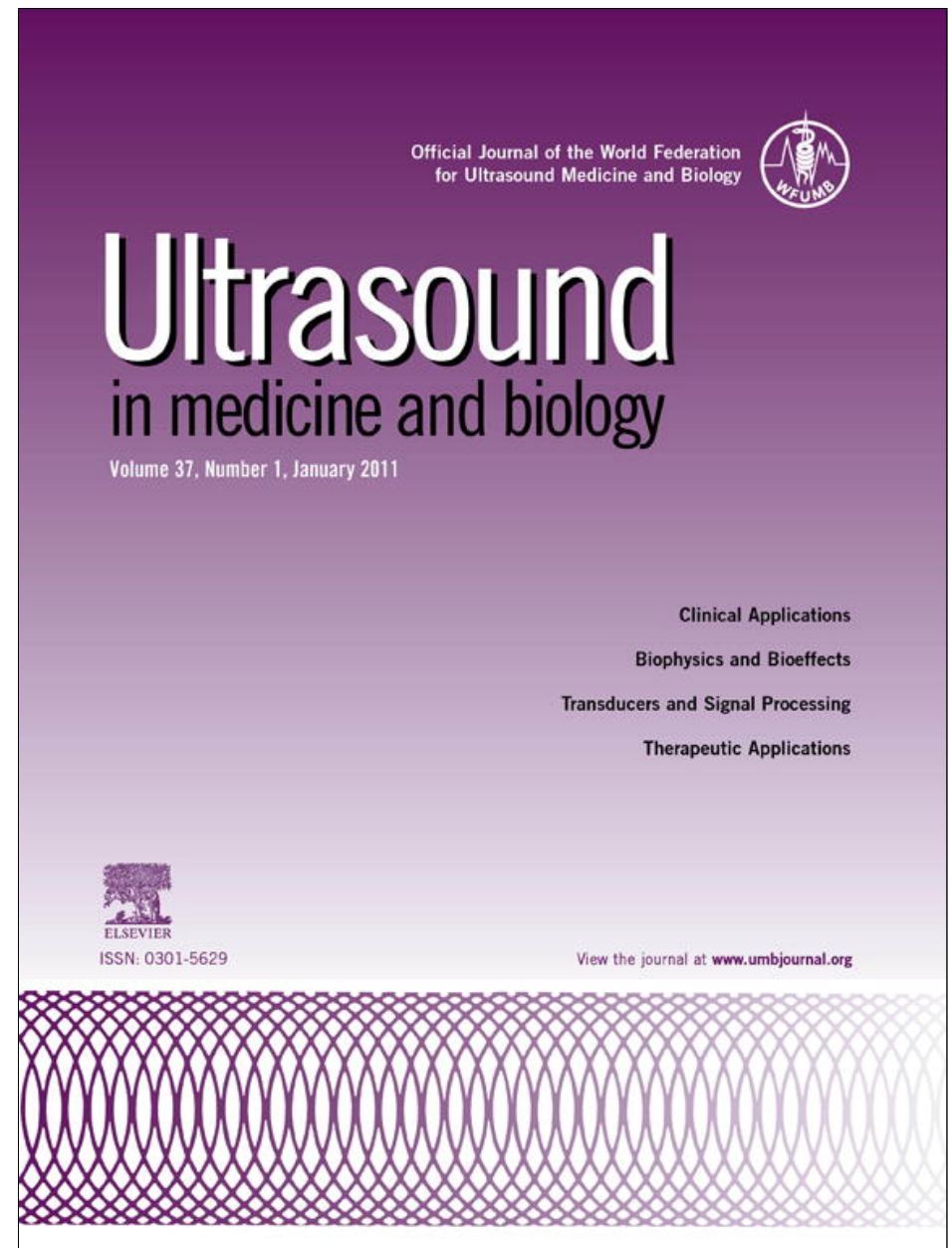

This article appeared in a journal published by Elsevier. The attached copy is furnished to the author for internal non-commercial research and education use, including for instruction at the authors institution and sharing with colleagues.

Other uses, including reproduction and distribution, or selling or licensing copies, or posting to personal, institutional or third party websites are prohibited.

In most cases authors are permitted to post their version of the article (e.g. in Word or Tex form) to their personal website or institutional repository. Authors requiring further information regarding Elsevier's archiving and manuscript policies are encouraged to visit:

http://www.elsevier.com/copyright 


\title{
LIVER CONTRAST ENHANCED ULTRASOUND PERFUSION IMAGING IN THE EVALUATION OF CHRONIC HEPATITIS C FIBROSIS: PRELIMINARY RESULTS
}

\author{
Antonio Orlacchio, ${ }^{*}$ Francesca Bolacchi, ${ }^{*}$ Maria Chiara Petrella, ${ }^{*}$ Daniela Pastorelli, ${ }^{*}$ \\ Gabriele Bazzocchi, ${ }^{*}$ Mario Angelico, ${ }^{\dagger}$ and Giovanni Simonetti* \\ * Department of Diagnostic Imaging-Molecular Imaging-Interventional Radiology, and Radiation Therapy; and ${ }^{\dagger}$ Hepatology \\ Unit, Department of Internal Medicine, University Hospital Tor Vergata, Rome, Italy
}

(Received 13 February 2010; revised 1 October 2010; in final form 9 October 2010)

\begin{abstract}
We wanted to determine whether liver contrast-enhanced ultrasound (CEUS)-derived peak signal intensity (PSI) and peak signal intensity/time (PIT) predict liver fibrosis in chronic hepatitis C (CHC). Fortynine patients with CHC (METAVIR classification) and 10 control subjects were included in the study. After a bolus of $2.4 \mathrm{~mL}$ SonoVue (Bracco Imaging, Milan, Italy) solution was injected into a peripheral vein, the right lobe of the liver containing the right portal vein was scanned in a transverse section. Two-dimensional sonography was performed using the Philips iU22 ultrasound system (Philips Healthcare, Best, the Netherlands). A 1.0-5.0-MHz (C5-1) wideband convex transducer was used, applying the following settings in all cases. Regions of interest were manually drawn over the right liver lobe and over the portal vein (PV). Liver parenchyma PSI (LPpsi) and PIT (LPpit), portal vein PSI (PVpsi) and PIT (PVpit) were automatically calculated. $\delta$ PSI was defined as

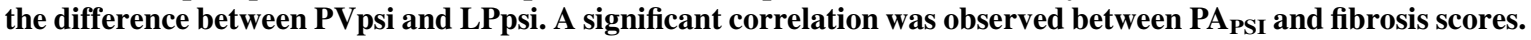
When patients were stratified according to their LPpsi, a significant difference was achieved only between patients with fibrosis score 0-1 vs. 2-3 and 2 vs. 4. Statistically significant differences between all fibrosis scores, except 0 vs. 1 and 3 vs. 4 were observed when $\delta$ PSI was used to stratify patients. Overall diagnostic accuracy of LPpsi and $\delta$ PSI measurement for severe fibrosis by area under the receiving operator characteristic curve analysis was, respectively, 0.87 and 0.88 . We suggest that liver CEUS perfusion could have the potential to be used as a complementary tool for the evaluation of liver fibrosis. However, further large-scale studies are required to accurately assess its accuracy in the evaluation of liver fibrosis. (E-mail: aorlacchio@uniroma2.it) @ 2011 World Federation for Ultrasound in Medicine \& Biology.
\end{abstract}

Key Words: Liver fibrosis, Perfusion imaging, Contrast-enhanced ultrasound, Chronic hepatitis C.

\section{INTRODUCTION}

Chronic hepatitis $\mathrm{C}(\mathrm{CHC})$ is characterized by progressive liver fibrosis, which has been shown to be a bidirectional process. A growing body of evidences from laboratory and clinical studies has shown that fibrosis is a potentially reversible process under specific therapies (Fowell and Iredale 2006). Therefore, the need for an accurate noninvasive test to monitor liver fibrosis is essential. Noninvasive markers have been shown to be insufficiently reliable (Sebastiani et al. 2008). Thus, liver biopsy remains currently the "gold standard" in the assessment of diffuse liver disease severity. However,

Address correspondence to: Antonio Orlacchio, Department of Diagnostic Imaging-Molecular Imaging-Interventional Radiology, and Radiation Therapy, University Hospital Tor Vergata, Rome, Italy. E-mail: aorlacchio@uniroma2.it several clinical drawbacks are associated with its use. It is an invasive procedure, and it is prone to complications, some minor such as pain, and others more severe, with a recorded risk of death of $0.01 \%$ (Cadranel et al. 2000).

Changes in hepatic hemodynamics have been widely documented in liver fibrosis (Hirata et al. 2001). Contrast-enhanced ultrasonography (CEUS) perfusion imaging permits tissue hemodynamics imaging. Indeed, CEUS-derived time-acoustic intensity curves reflects the tissue-of-interest hemodynamics by measuring the changes of intensity of the harmonic frequencies versus time (Krix 2005). The majority of the previously published CEUS studies in patients with chronic diffuse liver disease places great emphasis on the hepatic artery-tovein time-acoustic intensity curves (Staub et al. 2009; Searle et al. 2008; Ridolfi et al. 2007; Lim et al. 2005; Blomley et al. 2003; Albrecht et al. 1999; Pedersen et al. 2005). However, there are limited data to date on 
liver CEUS perfusion imaging in patients with diffuse liver disease (Kaneko et al. 2005)

CEUS-related perfusion parameters include time to enhancement (ET), time to peak intensity (PIT), peak signal intensity (PSI), enhancement duration (ED) and area under the curve (AUC). Of the aforementioned parameters, PIT proved to be the most reliable parameter in perfusion studies in brain tissue comparing AUC, peak intensity (Harrer et al. 2004; . Meves et al. 2002). On the other hand, PSI has shown that there is a difference between normal liver and cirrhosis in a study performed using a first-generation ultrasound contrast medium (Kaneko et al. 2005).

SonoVue (Bracco Imaging, Milan, Italy) is an intravascular ultrasound contrast agent that consists of stabilized microbubbles of a sulfur hexafluoride gas (Leen et al. 2002). Experimental evidence in rats has shown that microbubble agents containing perfluorocarbon gas, such as SonoVue, enhance the healthy liver by virtue of its slow flow within the sinusoids (Kono et al. 2002).

Based on the fact that sinusoidal volume of the hepatic parenchyma is decreased in liver fibrosis (Rockey et al. 2001), the purpose of the present study was to correlate the liver SonoVue CEUS perfusionderived parenchyma and portal vein PSI and PIT with histology in CHC patients stratified according to their liver histological fibrosis score.

\section{PATIENTS AND METHODS}

\section{Patients}

We prospectively enrolled a sample of 49 nonconsecutive $\mathrm{CHC}$ patients ( 27 male and 22 female; mean age $68 \mathrm{y}$, range 57 to 72 ) presenting with different fibrosis stages. Nine patients were staged F0, 11 patients F1, 10 patients F2, 12 patients F3 and 7 patients F4. Inclusion criteria were the presence of anti-hepatitis $\mathrm{C}$ virus (HCV) antibodies detected by third-generation test (enzyme-linked immunosorbent assay, recombinant-based immunoblot assay; Abbott, Pomezia, Italy); detectable serum HCVRNA; and liver biopsy findings compatible with $\mathrm{CHC}$. Patients with chronic hepatitis B and autoimmune hepatitis, or any cardiac or vascular dysfunction, were excluded. Liver biopsies were performed within the prior five months before ultrasound analysis. Ten healthy volunteers that matched the enrolled patients in age and sex were taken as controls. All controls had no history of liver disease, alcoholism, blood transfusion, positive tests for anti-HCV, anti-hepatitis B virus (HBV) or anti-human immunodeficiency virus (HIV) antibodies. At the time of ultrasound analysis none, of the subjects had fever or evidence of infectious or inflammatory disorders or any other kind of malignancies. All subjects involved in the study were kept fasted for the $8 \mathrm{~h}$ before the CEUS examination. The study was approved by our internal committee, and all patients and controls gave written informed consent.

\section{Histological evaluation}

Liver biopsy specimens $>10 \mathrm{~mm}$ in length were fixed in formalin, paraffin-embedded and stained with hematoxylin-eosin or picro Sirius red for collagen, and Perls' technique for iron. All biopsies were performed in the right hepatic lobe, where the sample for the CEUS acquisitions was located. For each liver biopsy specimen, histological fibrosis was scored according to the METAVIR classification (Goodman 2007). Fibrosis was staged on a $0-4$ scale: 0 , no fibrosis; 1 , portal fibrosis without septa; 2 , few septa; 3 , numerous septa without cirrhosis; 4, cirrhosis.

\section{CEUS perfusion imaging}

Two-dimensional sonography was performed using the Philips iU22 ultrasound system (Philips Healthcare) by a single radiologist with 10 years of experience in ultrasound imaging, blinded to the pathological changes of liver. A 1.0-5.0-MHz (C5-1) wideband convex transducer (Philips Healthcare) was used, applying the following settings in all cases: Mean depth $16 \mathrm{~cm}$, mean angle of view $60^{\circ}$, mechanical index 0.08 , overall gain set to obtain a complete anechoic image of the liver parenchyma for the basal phase, dynamic range set to 38 . The right lobe of the liver was scanned in a transverse section containing the right portal vein. Patients were trained to perform light breathing to minimize movement artifacts. A bolus of $2.4 \mathrm{~mL}$ SonoVue solution (sulfur hexafluoride) was injected into a peripheral vein using a 20-gauge venous catheter. Because preliminary experiments showed no obvious difference between the injection of 5 and $2.4 \mathrm{~mL}$ of SonoVue, the standard dose in this study had been defined as $2.4 \mathrm{~mL}$. The SonoVue injection was followed immediately by a $5-\mathrm{mL}$ saline bolus to flush the injection line. During the examination, the transducer was held in a fixed position (total scan time $3 \mathrm{~min}$ ). All examinations were stored on CD-ROM.

Liver perfusion imaging was evaluated on video. To detect the low signals from the contrast media, a standard preset on the US system was used. The standard preset used helped us study the intensity of the contrast medium excluding the contribution of the liver parenchyma basal signal. Thus, the intrapatient variability was minimized and more reproducible results were obtained.

\section{Data processing}

The time-acoustic intensity curves were analyzed automatically by the quantitative analysis software package QLAB (Philips Healthcare). A region of interest (ROI) of $600 \mathrm{~mm}^{2}$ was manually drawn over the right 
liver lobe (LP), whereas a ROI of $100 \mathrm{~mm}^{2}$ was placed over the portal vein (PV) (Fig. 1). Attention was paid not to include vessels in the $600 \mathrm{~mm}^{2}$ ROI. Movement artifacts were eliminated in the post-processing by deleting selected frames. A gamma variate fit (a statistical model used to normalize the dispersion of gamma values in a perfusion analysis study) of the LP and PV intensity curves was performed and both liver parenchyma and portal vein PSI and PIT were automatically calculated. PIT was defined as the time period between the initial time (contrast medium injection) and the time of signal intensity peak. $\delta$ PSI was defined as the difference between the PSI of the PV and the PSI of the LP measured from the time-acoustic intensity curve. Uncompressed linear data (raw data) analysis was performed in consensus by two radiologists with, respectively, 4 and 10 years of experience in ultrasound imaging, both blinded to the pathological changes of the liver.

\section{Statistical analysis}

Data were analyzed using the nonparametric MannWhitney $U$ test. Correlations were evaluated with the Spearman nonparametric test. A $p$-value $<0.05$ was considered significant. Data were expressed as medians \pm the interquartile range (IQR) for each group. The diagnostic value of SonoVue CEUS liver perfusion measurement relative to histological fibrosis staging (METAVIR score, F0 to F4) was determined on the basis of assessment of sensitivity, specificity and was determined by varying the threshold values and constructing receiver operating characteristic (ROC) curves. The comparison was made to distinguish significant fibrosis (METAVIR score, F2, F3, F4) from no or mild fibrosis (METAVIR score, F0 and F1). For this comparison, examination of a biopsy specimen was considered to be the gold

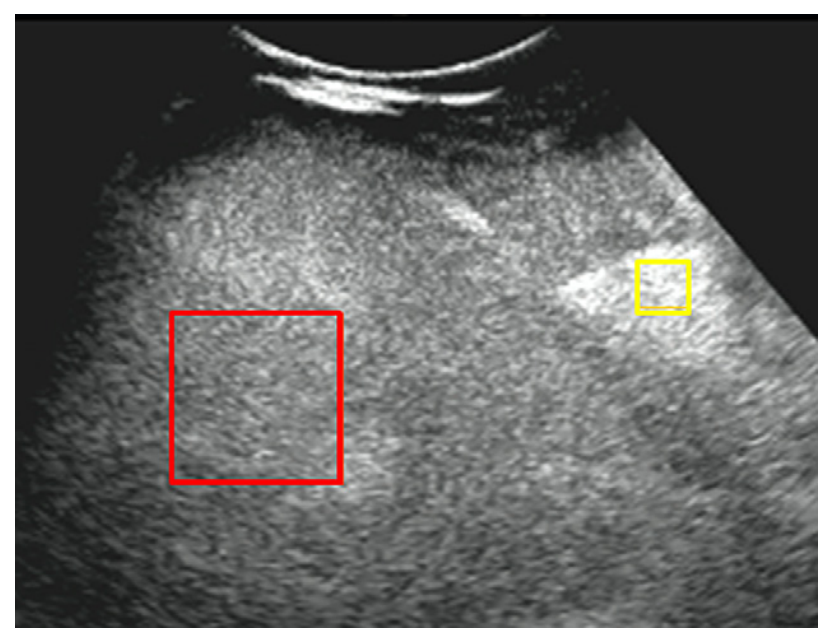

Fig. 1. Image shows ROI positioning over the right liver lobe (largest square) and over the portal vein (smallest square) in a 65 -year-old woman with fibrosis score 2. standard. We present area-under-the-ROC curve (AUCROC) as a global measure of SonoVue CEUS liver perfusion measurement accuracy. Optimal SonoVue liver perfusion measurement cutoff value for classification of the dichotomous histological outcomes was determined by maximizing the combination of sensitivity and specificity. The method of Hanley and McNeil was used for the calculation of the difference between AUCs.

Statistical analysis was performed using commercially available software (GraphPad v. 5; GraphPad Software, La Jolla, CA, USA, and MedCalc v. 11.3.3; MedCalc Software, Mariakerke, Belgium).

\section{RESULTS}

CEUS time-acoustic intensity curves of LP and PV were obtained in all patients. Analysis of the profile of the time acoustic intensity curve showed a rapid-rising slope from the time of the bolus injection to the PSI and a gradual descending slope after reaching PSI in

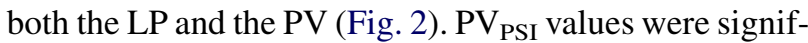
icantly higher than $\mathrm{LP}_{\mathrm{PSI}}$ values (median values, 44 [IQR, 41 to 48] vs. 29 [IQR, 24 to 33], $p<0.001$ ]). As shown in Fig. 3, there was no statistically significant correlation between PVpsi and the fibrosis scores $(\mathrm{r}=0.23,95 \%$ CI -0.04 to $0.48, p=0.09$ ). A significant correlation was observed between the $\mathrm{LP}_{\mathrm{PSI}}$ values and the fibrosis scores $(\mathrm{r}=-0.72,95 \% \mathrm{CI}=-0.84$ to $-0.56, p<$ 0.001) (Fig. 4). A significant difference in $\mathrm{LP}_{\mathrm{PSI}}$ was achieved between patients with fibrosis score 1 vs. 2 and 2 vs. 4 (LPPSI median values, 32 [IQR, 28 to 36] vs. 26 [IQR, 24 to 30], $p=0.033$ and 28 [27 to 30) vs. 21 [19 to 25$], p=0.001)$. When $\delta$ PSI was used to stratify
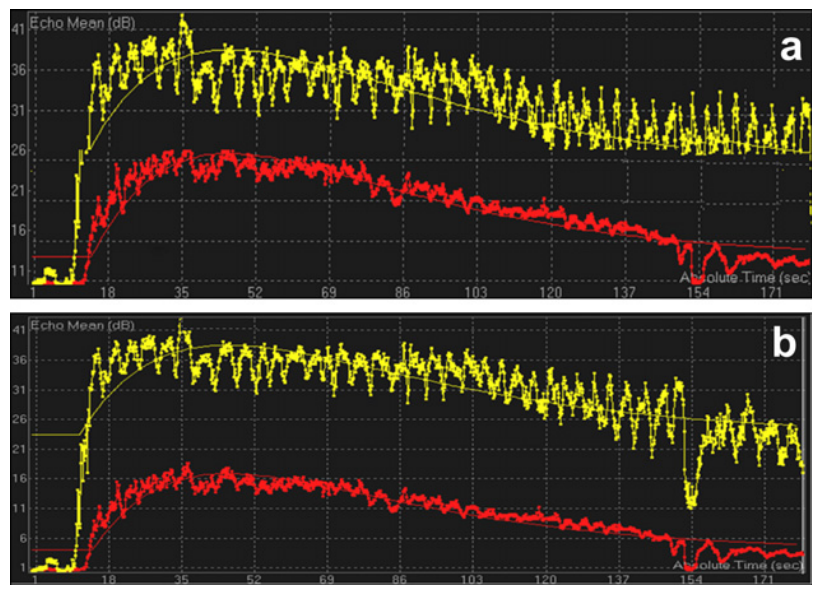

Fig. 2. (a-b) The time-acoustic intensity curves of 64-year-old woman with fibrosis score 1 (a) and a 70-year-old man with fibrosis score 3 (b). In the upper position is the PV curve, and in the lowest position the liver parenchyma curve. Echo mean is the mean echo intensity values in the selected ROI. Absolute time is the time course analysis. 


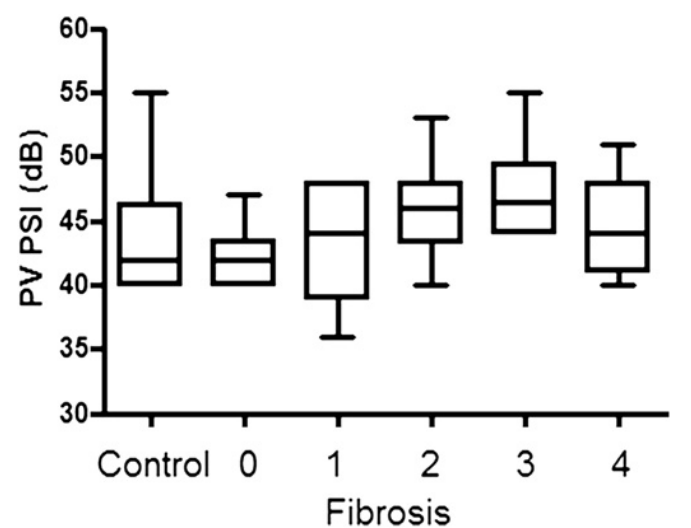

Fig. 3. Correlation between $\mathrm{PV}_{\mathrm{PSI}}$ with fibrosis scores. Values are median, range and interquartile range $(p=0.19)$.

patients as shown in Fig. 5, a significant difference was observed between fibrosis score 1 vs. 2 and 2 vs. 3 (median values, 12 [IQR 8 to 16] vs. 17 [IQR, 12 to 19], $p=0.022 ; 17$ [IQR, 12 to 19] vs. 20 [IQR 17 to $22], p=0.031)$. $\mathrm{PV}_{\mathrm{PIT}}$ were significantly higher than $\mathrm{LP}_{\mathrm{PIT}}$ values (median values 44 [IQR, 41 to 48$]$ vs. 35 [IQR, 31 to 38], $p<0.001)$. However, no correlations were observed between $\mathrm{LP}_{\mathrm{PIT}}$ or $\mathrm{PV}_{\mathrm{PIT}}$ and fibrosis severity $\left(\mathrm{PV}_{\mathrm{PIT}}: \mathrm{r}=0.03,95 \% \mathrm{CI}=-0.02\right.$ to $0.11, p=$ 0.83 ; LP $\mathrm{PIT}_{\mathrm{PI}} \mathrm{r}=0.04,95 \% \mathrm{CI}=-0.03$ to $0.13, p=$ 0.64). The diagnostic accuracy of $\delta$ PSI measurement for severe fibrosis by AUC-ROC analysis was 0.87 (95\% CI, 0.74-0.94). At a cutoff of $>16, \delta$ PSI showed a specificity of $83 \%$ and a sensitivity of $77 \%$ (Fig. 6a). Overall diagnostic accuracy of LPpsi for severe fibrosis calculated for by AUC-ROC analysis was 0.88 (CI 0.760.95 ). At a cut-off of $\leq 31$, LPpsi showed a specificity of $70 \%$ and a sensitivity of $95.5 \%$ (Fig. 6b). No statistical significant differences were observed between $\delta$ PSI and LPpsi AUCs (z statistics 0.29, $p=0.82$ ) (Fig. 7).

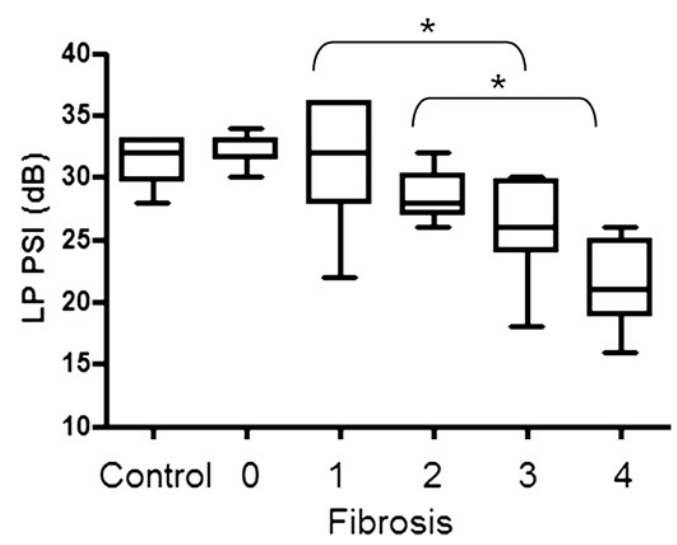

Fig. 4. Correlation between $\mathrm{LP}_{\mathrm{PSI}}$ with fibrosis scores. Values are median, range and interquartile range $\left({ }^{*} p<0.05\right)$.

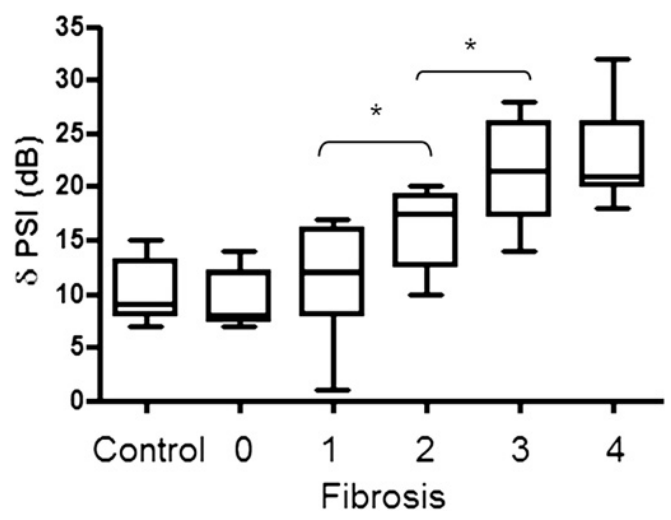

Fig. 5. Correlation between $\delta \mathrm{PSI}\left(\mathrm{PV}_{\mathrm{PSI}}-\mathrm{LP}_{\mathrm{PSI}}\right)$ with fibrosis scores. Values are median, range and interquartile range $(* p<0.05)$.

\section{DISCUSSION}

CEUS perfusion, evaluating the blood flow per tissue and time unit including capillary flow, is an important functional tool for the evaluation of tissue pathological processes (Weber et al. 2007). Kaneko et al. (2005) showed a significant negative relationship between the gray-scale intensity of nontumorous areas of liver parenchyma in normal liver tissue versus cirrhosis and in chronic hepatitis versus cirrhosis. They demonstrated a significant difference between a normal and cirrhotic liver. However, they did not stratify the patients according to their liver disease severity (Kaneko et al. 2005). Here, we correlated the liver CEUS perfusion derived parenchyma and PV PSI and PIT with histology in CHC patients stratified according to their liver histological fibrosis score. In accordance with previously published data by Li et al. (2006), our data showed that PV $\mathrm{PSI}_{\text {was }}$ constantly higher than LP PSI $_{\text {Palues in all fibrosis }}$ stages. In our study, we found no correlation between $\mathrm{PV}_{\mathrm{PSI}}$ values and fibrosis scores. On the contrary, we found that $\mathrm{LP}_{\mathrm{PSI}}$ decreased progressively with disease severity as determined by the histopathologic fibrosis scores. When patients were stratified according to their $\mathrm{LP}_{\mathrm{PSI}}$, a significant difference was achieved between patients with fibrosis score $0-1 v s$. patients with fibrosis score 2-3 and 2 vs. 4 . When patients were stratified according to their difference between their PV and their $\mathrm{LP}_{\mathrm{PSI}}$, i.e., to their $\delta \mathrm{PSI}$, a significant difference was observed between fibrosis score 1 vs. 2 and 2 vs. 3. No differences were observed between control subjects and patients with fibrosis score 0 or 1 and between fibrosis 3 and 4 in any case. Overall diagnostic accuracy of LP $P_{P S I}$ and $\delta$ PSI measurement for severe fibrosis measured by AUCs using ROC analysis were, respectively, 0.87 and 0.88 .

Our data are in accordance with Zhang and Cao (2007), who evaluated a new self-made contrast agent in 

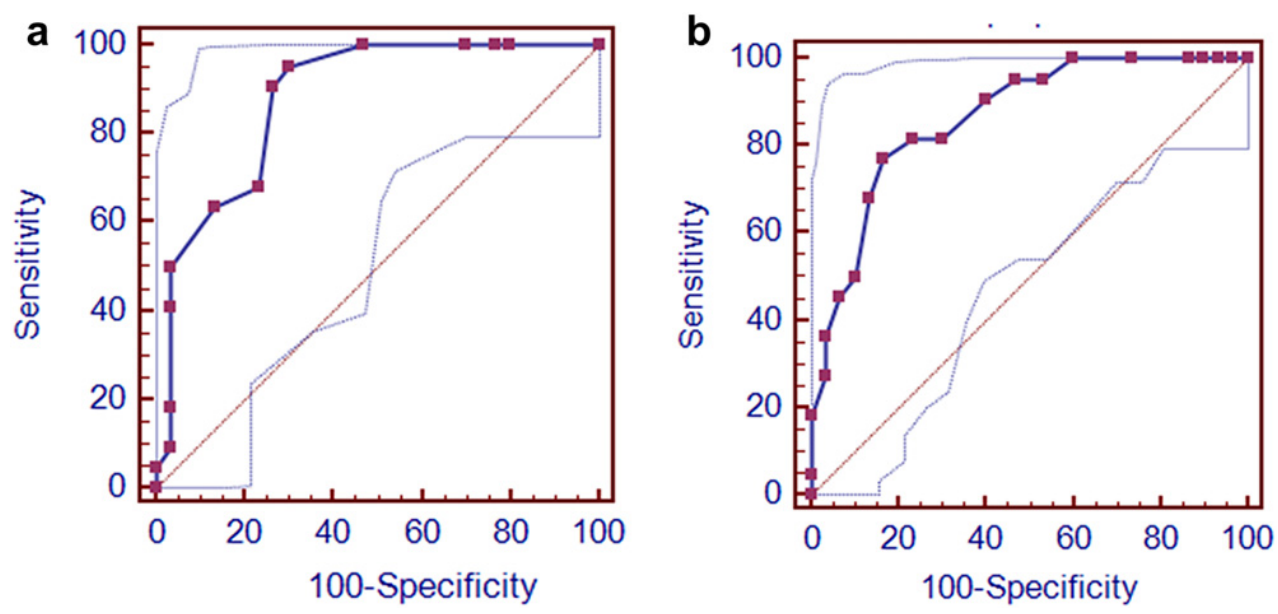

Fig. 6. (a-b) AUC-ROC graphs show diagnostic accuracy for severe fibrosis of $\mathrm{LP}_{\mathrm{PSI}}(\mathrm{a})$ and $\delta \mathrm{PSI}$ (b).

a rabbit liver fibrosis model. In our study, we used SonoVue, a second-generation ultrasound contrast agent. SonoVue is an intravascular agent (Leen et al. 2002) and the mechanism underlying liver parenchymal enhancement remains unknown (Leen et al. 2006). It has been suggested that the persistence of the agent within the healthy liver may be a result of the very slow flow within the sinusoids (Leen et al. 2006). Indeed, experimental evidence in rats has shown that microbubble agents containing perfluorocarbon gas enhance the healthy liver by virtue of the slow flow within the sinusoids (Kono et al. 2002). Because hepatic fibrosis decreases the sinusoidal volume of the hepatic parenchyma (Rockey 2001), the anatomical changes of the sinusoids occurring in a fibrotic liver could be considered as a possible explanation for the decrease in $\mathrm{LP}_{\mathrm{PSI}}$ and in $\mathrm{PV}_{\mathrm{PSI}}$ always being constantly higher as observed in our study.

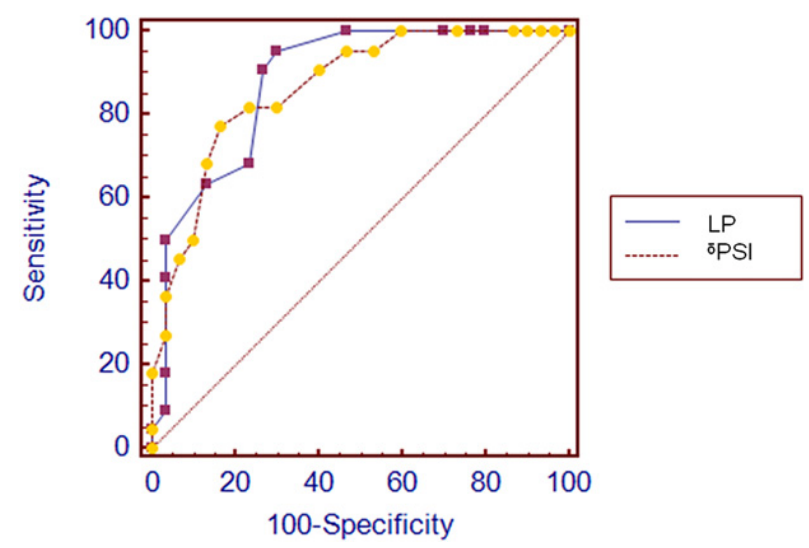

Fig. 7. Graph shows comparison between LPPSI and $\delta$ PSI ROC curves. No significant statistical difference was observed between the two curves $(p=0.82)$.
There were some limitations in the present study. First, the study population was relatively small and this is a preliminary study that needs further experiences. Also, we have not evaluated the inter- and intraobserver agreement of the method described, which are necessary to use the method to follow individual patients. Similarly, technique reproducibility was not evaluated. Additional studies with larger cohorts of patients are required to further establish interobserver variability and to assess intraobserver variability and reproducibility of the method.

In conclusion, we suggest that liver CEUS perfusion imaging could have the potential to be used as a complementary tool for the evaluation of liver fibrosis. However, further larger-scale studies are required to assess the real boundaries of accuracy of the test. If our results will be confirmed, CEUS will be an easy and valuable noninvasive method for diagnosis and assessment of liver fibrosis.

Acknowledgments-The authors thank Sandro Stefanelli (Philips Medical Systems) for the help in data acquisition.

\section{REFERENCES}

Albrecht T, Blomley MJ, Cosgrove DO, Taylor-Robinson SD, Jayaram V, Eckersley R, Urbank A, Butler-Barnes J, Patel N. Non-invasive diagnosis of hepatic cirrhosis by transit-time analysis of an ultrasound contrast agent. Lancet 1999;353:1579-1583.

Blomley MJ, Lim AK, Harvey CJ, Patel N, Eckersley RJ, Basilico R, Heckemann R, Urbank A, Cosgrove DO, Taylor-Robinson SD. Liver microbubble transit time compared with histology and Child-Pugh score in diffuse liver disease: A cross sectional study. Gut 2003; 52:1188-1193.

Cadranel JF, Rufat P, Degos F. Practices of liver biopsy in France: Results of a prospective nationwide survey. For the Group of Epidemiology of the French Association for the Study of the Liver (AFEF). Hepatology 2000;32:477-481.

Fowell AJ, Iredale JP. Emerging therapies for liver fibrosis. Dig Dis 2006;24:174-183. 
Goodman ZD. Grading and staging systems for inflammation and fibrosis in chronic liver diseases. J Hepatol 2007;47:598-607.

Harrer JU, Klotzsch C, Stracke CP, Möller-Hartmann W. Cerebral perfusion sonography in comparison with perfusion MRT: A study with healthy volunteers. Ultraschall Med 2004;25:263-269.

Hirata M, Akbar SM, Horiike N, Onji M. Noninvasive diagnosis of the degree of hepatic fibrosis using ultrasonography in patients with chronic liver disease due to hepatitis $\mathrm{C}$ virus. Eur J Clin Invest 2001;31:528-535.

Kaneko T, Teshigawara O, Sugimoto H, Hirota M, Inoue S, Takeda S, Nakao A. Signal intensity of the liver parenchyma in microbubble contrast agent in the late liver phase reflects advanced fibrosis of the liver. Liver Int 2005;25:288-293.

Kono Y, Steinbach GC, Peterson T, Schmid-Schönbein GW, Mattrey RF. Mechanism of parenchymal enhancement of the liver with a microbubble-based US contrast medium: An intravital microscopy study in rats. Radiology 2002;224:253-257.

Krix M. Quantification of enhancement in contrast ultrasound: A tool for monitoring of therapies in liver metastases. Eur Radiol 2005;15: 104-108.

Leen E, Angerson WJ, Yarmenitis S, Bongartz G, Blomley M, Del Maschio A, Summaria V, Maresca G, Pezzoli C, Llull JB. Multicentre clinical study evaluating the efficacy of SonoVue (BR1), a new ultrasound contrast agent in Doppler investigation of focal hepatic lesions. Eur J Radiol 2002;41:200-206.

Leen E, Ceccotti P, Kalogeropoulou C, Angerson WJ, Moug SJ, Horgan PG. Prospective multicenter trial evaluating a novel method of characterizing focal liver lesions using contrast-enhanced sonography. AJR Am J Roentgenol 2006;186:1551-1559.

Li J, Dong BW, Yu XL, Li CF. Gray scale contrast enhancement and quantification in different positions of rabbit liver. J Ultrasound Med 2006;25:7-14.

Lim AK, Taylor-Robinson SD, Patel N, Eckersley RJ, Goldin RD, Hamilton G, Foster GR, Thomas HC, Cosgrove DO, Blomley MJ. Hepatic vein transit times using a microbubble agent can predict disease severity non-invasively in patients with hepatitis C. Gut 2005;54:128-133.

Meves SH, Wilkening W, Thies T, Eyding J, Hölscher T, Finger M, Schmid G, Ermert H, Postert T; Ruhr Center of Competence for Medical Engineering. Comparison between echo contrast agentspecific imaging modes and perfusion-weighted magnetic resonance imaging for the assessment of brain perfusion. Stroke 2002;33: 2433-2437.

Pedersen JF, Larsen VA, Bytzer P, Madsen LG, Hamberg O. Hepatic transit time of ultrasound contrast in biopsy characterized liver disease. Acta Radiol 2005;46:557-560.

Ridolfi F, Abbattista T, Marini F, Vedovelli A, Quagliarini P, Busilacchi P, Brunelli E. Contrast-enhanced ultrasound to evaluate the severity of chronic hepatitis C. Dig Liver Dis 2007;39:929-935.

Rockey D. Hepatic blood flow regulation by stellate cells in normal and injured liver. Semin Liver Dis 2001;21:337-350.

Sebastiani G, Vario A, Guido M, Alberti A. Performance of noninvasive markers for liver fibrosis is reduced in chronic hepatitis $\mathrm{C}$ with normal transaminases. J Viral Hepat 2008;15:212-218.

Searle J, Mendelson R, Zelesco M, Sanford J, Cheng W, McKinstry C, Ramsay D. Non-invasive prediction of the degree of liver fibrosis in patients with hepatitis $\mathrm{C}$ using an ultrasound contrast agent. A pilot study. J Med Imaging Radiat Oncol 2008;52:130-133.

Staub F, Tournoux-Facon C, Roumy J, Chaigneau C, MorichautBeauchant M, Levillain P, Prevost C, Aubé C, Lebigot J, Oberti F, Galtier JB, Laumonier H, Trillaud H, Bernard PH, Blanc JF, Sironneau S, Machet F, Drouillard J, de Ledinghen V, Couzigou P, Foucher P, Castéra L, Tranquard F, Bacq Y, d'Altéroche L, Ingrand P, Tasu JP. Liver fibrosis staging with contrast-enhanced ultrasonography: Prospective multicenter study compared with METAVIR scoring. Eur Radiol 2009;19:824-829.

Weber MA, Krix W, Delorme S. Quantitative evaluation of muscle perfusion with CEUS and with MR. Eur Radiol 2007; 17:2663-2674.

Zhang Y, Cao TS. Grey scale enhancement by a new self-made contrast agent in early cirrhotic stage of rabbit liver. BMC Gastroenterol 2007;8:7-32. 